\title{
Guest Editorial: Security Challenges in the loT Regime
}

\author{
Sandip Ray ${ }^{1} \cdot$ Yier $\operatorname{Jin}^{2}$ \\ Received: 14 November 2017 / Accepted: 17 November 2017 / Published online: 12 December 2017 \\ (C) Springer International Publishing AG, part of Springer Nature 2017
}

The era of Internet-of-Things (IoT) is characterized by an environment in which we live in an ecosystem consisting of billions of smart, connected computing devices. Most of these devices are equipped with complex sensors that continuously collecting, analyzing, and communicating data pertaining to some of our most private and intimate activities, including sleep, health, location, social contacts, and browsing patterns. Security and privacy are clearly crucial to computing devices in this era. Security issues can come at different levels, including deployment issues that leave devices unprotected from cyber-attack, functional vulnerabilities in system design or implementation, an altered functionality by some player in the complex supply-chain, or side-channel issues exploitable by physical access to the device. Furthermore, data collected by these devices have a complex communication path through switches, gateways, routers, and the cloud of servers and datacenters. Vulnerabilities in any point of this communication can compromise the entire IoT infrastructure and have catastrophic consequences.

This HaSS Special Issue focuses on research challenges in ensuring security and trustworthiness in computing devices in the IoT regime, where the notion of "computing devices" is interpreted broadly to include not only sensor-attached "edge" devices but also to routers, gateways, and datacenters in contexts where they pertain to an IoT infrastructure. The aim is to provide a spectrum of challenges, approaches, and solutions, and provide an authoritative reference of the state of the art in security and trustworthiness issues for IoT.

Obviously, it is beyond the scope of a Special Issue to provide a comprehensive treatment of this vast area. Instead, we selected six representative papers to provide

Sandip Ray

sandip.ray@nxp.com

NXP Semiconductors, Austin, TX 78735, USA

2 Department of Electrical and Computer Engineering, University of Florida, Gainesville, FL 32611, USA a sampling of the various facets of research challenges in this area.

(1) "Attacks in Reality: The Limits of Concurrent Error Detection Codes against Laser Fault Injection," by J. Breier et al.,

(2) "TSensors Vision, Infrastructure and Security Challenges in Trillion Sensor Era: Current Trends and Future Directions," by M. Alam et al.,

(3) "Replacing eFlash with STTRAM in IoTs: Security Challenges and Solutions," by A. De et al.,

(4) "Reducing Side Channel Leakage of Encryption Engines using Integrated Low-dropout Voltage Regulators," by A. Singh et al.,

(5) "Cross-level Detection Framework for Attacks on Cyber-Physical Systems," by B. Croteau et al., and

(6) "Internet-of-Things Security and Vulnerabilities: Taxonomy, Challenges, and Practice," by K. Chen et al.

The last paper from the above list is a summary article written by the Guest Editors and their students reflecting on the spectrum and scope of IoT security. Since a key goal of the Special Issue is to provide a comprehensive reference for the different research challenges and progress, each article includes a detailed discussion of related research.

We express our sincere thanks to all the authors and referees for their contribution in creating this special issue. We thank the Editors-in-Chief for their encouragement and support (and friendly nudges at various stages of the process), and the administrative staff for technical help in this long process. We hope you enjoy this issue and that it inspires more research to identify and overcome future security challenges in our increasingly connected world.

Yier Jin and Sandip Ray

Guest Editors

Journal of Hardware and Systems Security (HaSS) 